\title{
Efficacy of immune checkpoint inhibitors in non-small cell lung cancer with uncommon histology: a propensity-score-matched analysis
}

Koichi Miyashita', Masato Karayama ${ }^{1 *}$, Yusuke Inoue ${ }^{1}$, Hironao Hozumi ${ }^{1}$, Yuzo Suzuki ${ }^{1}$, Kazuki Furuhashi $^{1}$, Tomoyuki Fujisawa' , Noriyuki Enomoto' ${ }^{1}$ Yutaro Nakamura ${ }^{1,2}$, Masato Kono ${ }^{3}$, Takashi Matsui ${ }^{4}$, Mitsuru Niwa ${ }^{5}$, Keigo Koda ${ }^{6}$, Mikio Toyoshima ${ }^{6}$, Sayomi Matsushima ${ }^{7}$, Shun Matsuura ${ }^{8}$, Kazuhiro Asada ${ }^{9}$, Masato Fujii ${ }^{10}$, Hideki Kusagaya ${ }^{11}$, Hiroyuki Matsuda ${ }^{12}$, Naoki Inui ${ }^{13}$ and Takafumi Suda ${ }^{1}$

\begin{abstract}
Background: Clinical efficacy of immune checkpoint inhibitors (ICls) for non-small cell lung cancer (NSCLC) with uncommon histology (UNSCLC) is unknown.

Methods: Patients with NSCLC treated with ICI monotherapy between January 2014 and December 2018 in 10 Japanese hospitals were retrospectively evaluated. The patients were divided into: (1) NSCLC with common histology (cNSCLC), defined as adenocarcinoma and squamous cell carcinoma; and (2) UNSCLC, defined as incompatibility with morphological and immunohistochemical criteria for adenocarcinoma or squamous cell carcinoma. Propensity score matching was performed to balance the two groups.
\end{abstract}

Results: Among a total of 175 patients included, 44 with uNSCLC (10 pleomorphic carcinomas, 9 large cell neuroendocrine carcinomas, 2 large cell carcinomas, and 23 not otherwise specified) and 44 with matched CNSCLC (32 adenocarcinomas and 12 squamous cell carcinomas) were selected for analyses. Median progression-free survival (PFS) (4.4 months, 95\% confidence interval [CI] 1.8-7.7 months) and overall survival (OS) (11.4 months, 95\% Cl 7.4-27.4 months) in the UNSCLC patients were not significantly different from those in matched CNSCLC patients $(5.4$ months, $95 \% \mathrm{Cl}$ 3.1-7.6 months, $p=0.761$; and 14.1 months, $95 \% \mathrm{Cl} 10.6-29.6$ months, $p=0.381)$. In multivariate analysis, Eastern Cooperative Oncology Group performance status (ECOG-PS) of 0-1 and programmed death ligand-1 (PD-L1) expression were predictive for PFS and OS in UNSCLC.

Conclusions: ICls had similar clinical efficacy for treatment of UNSCLC and CNSCLC. Good ECOG-PS and PD-L1 expression were predictive for efficacy of ICIs in UNSCLC.

Keywords: Pleomorphic carcinoma, Large cell neuroendocrine carcinoma, Not otherwise specified, Programmed death-1, Programmed death ligand-1

\footnotetext{
*Correspondence: karayama@hama-med.ac.jp

${ }^{1}$ Second Division, Department of Internal Medicine, Hamamatsu

University School of Medicine, 1-20-1 Handayama, Hamamatsu 431-3192,

Japan

Full list of author information is available at the end of the article
}

\begin{abstract}
Introduction
The emergence of immune checkpoint inhibitors (ICIs) has led to major changes in treatment paradigms for nonsmall cell lung cancer (NSCLC). Pembrolizumab, an antiprogrammed death-1 (PD-1) antibody, or atezolizumab, an anti-programmed death ligand-1 (PD-L1) antibody, have demonstrated survival benefits over platinum-based
\end{abstract}


chemotherapy in chemo-naïve patients with NSCLC [1, 2]. In previously treated patients with NSCLC, pembrolizumab, atezolizumab and nivolumab (anti-PD-1 antibody) have demonstrated long-term survival benefits over docetaxel [3-6]. Clinical guidelines recommend ICIs as first- second- or later-line treatments for unresectable NSCLC [7-9].

Although adenocarcinoma and squamous cell carcinoma are the dominant tumor pathologies in NSCLC, $8-18 \%$ of patients have uncommon histology, such as pleomorphic carcinoma, large cell neuroendocrine carcinoma (LCNEC), large cell carcinoma and not otherwise specified (NOS) [10-13]. As well as distinct histological features, NSCLC with uncommon histology ( $u$ NSCLC) has different clinical courses and poor therapeutic responses and prognosis compared with NSCLC with common histology ( $c$ NSCLC), such as adenocarcinoma or squamous cell carcinoma. For example, pleomorphic carcinoma of the lung is reported to progress aggressively and to be refractory to chemotherapy, with an objective response rate (ORR) of $17 \%$ and progression-free survival (PFS) of 2 months [14]. Patients with NSCLC-NOS are reported to have a median PFS of 5.9 months after first-line platinum-based chemotherapy, which is shorter than 7.3 months in patients with adenocarcinoma [15]. Patients with LCNEC have better clinical benefit from small cell lung cancer (SCLC)-based chemotherapy, such as etoposide/platinum, compared with NSCLC-based chemotherapy, such as gemcitabine/platinum, pemetrexed/platinum and paclitaxel/platinum [16].

However, little is known about the therapeutic benefits of ICIs for $u$ NSCLCs. Some clinical trials for ICIs in NSCLC have included $u$ NSCLCs; however, the proportion of $u$ NSCLCs in the total study populations was only $2-7 \%[4,5,17,18]$. Given the distinct features and poor therapeutic responses to cytotoxic chemotherapy, it is unknown whether patients with $u$ NSCLC have similar clinical benefits from ICIs as those with $c$ NSCLC. In this multicenter retrospective study, we compared the efficacy of ICIs in patients with $u$ NSCLC or $c$ NSCLC using propensity-score-matched analysis. Additionally, we identified predictive factors for ICIs in patients with $u$ NSCLC.

\section{Materials and methods Study design}

This was a multicenter, retrospective cohort study that was approved by the Institutional Review Board of each participating institution. Patient consent was waved because it was a retrospective study. This study was registered with the University Hospital Medical Information Network (ID: UMIN000037777).

\section{Patients}

We retrospectively reviewed medical records of patients who were diagnosed with advanced or recurrent NSCLC between January 2014 and December 2018 in 10 hospitals in Japan. Patients with pathologically diagnosed NSCLC who received ICI monotherapy were included. Any lines of treatment were allowed if ICI monotherapy was administered. The recurrent stage was defined as recurrence after radical surgery and applicable for systemic therapy, but not for local therapy. Patients who received combination therapy with platinum-based chemotherapy and ICIs or had histories of previous ICI therapy were excluded. The patients were divided into 2 groups on the basis of pathological diagnosis: (1) $c$ NSCLC, patients with adenocarcinoma or squamous cell carcinoma; and (2) $u$ NSCLC, those without morphological and immunohistochemical criteria for adenocarcinoma or squamous cell carcinoma, such as pleomorphic carcinoma, large cell carcinoma, LCNEC or NOS. Pathological diagnosis was performed morphologically and immunologically at each institution.

\section{Data collection}

Clinical data, including age, sex, smoking history, pathology, PD-L1 tumor proportion score (TPS), cancer staging, Eastern Cooperative Oncology Group performance status (ECOG-PS), line of treatment, and type of ICI were obtained from the patients' medical records. The responses to ICI were evaluated in accordance with the Response Evaluation Criteria in Solid Tumors (RESIST) version 1.1 [19]. Disease control rate (DCR) was defined as complete response (CR) plus partial response (PR) plus stable disease, and ORR as CR plus PR. PFS and OS were calculated from the date of first administration of ICI.

\section{Propensity score matching}

To balance the baseline of the two groups, 1:1 propensity score matching was performed. Propensity scores were calculated using a logistic regression model and included the following variables: age, sex, smoking status, cancer stage, PD-L1 TPS, line of ICI, and ECOG-PS.

\section{Statistical analysis}

Fisher's exact test and Mann-Whitney $U$ test were used for categorical and continuous variables, respectively. Kaplan-Meier method and the log-rank tests were used for PFS and OS. Cox proportional hazards regression analysis was used to identify predictive variables for PFS and OS. Logistic regression analysis was used to identify predictive variables for ORR and DCR. Variables of $p<0.100$ in univariate analyses, pathology ( $u$ NSCLC vs. $c$ NSCLC), and PD-L1 expression 
were included for multivariate analyses. All values are expressed as median (range) or number (\%). A $p$ value $<0.05$ was considered significant. All statistical analyses were performed with EZR (Saitama Medical Center, Jichi Medical University, Saitama, Japan), which is a graphical user interface for $\mathrm{R}$ (The R Foundation for Statistical Computing, Vienna, Austria, version 2.13.0) [20].

\section{Results}

Patient characteristics

A total of 175 patients (44uNSCLCs and $131 c$ NSCLCs) were included in the study. Patient characteristics are shown in Table 1 . The $u$ NSCLC group had a median age of 66 years, and most patients were men (95\%), and most had a smoking history (95\%) and good ECOG-PS of 0-1 (82\%). Eight (18\%), 10 (23\%), and 16 (36\%) patients in the $u$ NSCLC group had brain, liver and bone metastases,

Table 1 Patient characteristics

\begin{tabular}{|c|c|c|c|c|}
\hline & $\begin{array}{l}\text { Unmatched cNSCLC } \\
(n=131)\end{array}$ & $\begin{array}{l}\text { Matched cNSCLC } \\
(n=44)\end{array}$ & $\begin{array}{l}\text { uNSCLC } \\
(n=44)\end{array}$ & $p$-value \\
\hline Age, years & $69(43-83)$ & $67(44-81)$ & $66(40-83)$ & 0.923 \\
\hline Sex, men & $103(79)$ & $42(95)$ & $42(95)$ & 1.000 \\
\hline Smoking status, ever-smokers & $111(85)$ & $42(95)$ & $42(95)$ & 1.000 \\
\hline EGOG-PS, & & & & 0.237 \\
\hline 0 & $69(53)$ & $21(48)$ & $14(32)$ & \\
\hline $58(44)$ & $19(43)$ & $22(50)$ & & \\
\hline$\geq 2$ & $4(3)$ & $4(9)$ & $8(18)$ & \\
\hline Stage, & & & & 0.715 \\
\hline III & $23(18)$ & $14(32)$ & $14(32)$ & \\
\hline IV & $97(74)$ & $24(55)$ & $21(48)$ & \\
\hline Recurrence & $11(8)$ & $6(14)$ & $9(20)$ & \\
\hline \multicolumn{5}{|l|}{ Metastases, } \\
\hline Brain & $26(20)$ & $14(32)$ & $8(18)$ & 0.218 \\
\hline Liver & $13(10)$ & $4(9)$ & $10(23)$ & 0.143 \\
\hline Bone & $30(23)$ & $11(25)$ & $16(36)$ & 0.355 \\
\hline Pathology, & & & & $<0.001$ \\
\hline Adenocarcinoma & $85(65)$ & $32(73)$ & $0(0)$ & \\
\hline Squamous cell carcinoma & $46(35)$ & $12(27)$ & $0(0)$ & \\
\hline Pleomorphic carcinoma & $0(0)$ & $0(0)$ & $10(23)$ & \\
\hline LCNEC & $0(0)$ & $0(0)$ & $9(20)$ & \\
\hline Large cell carcinoma & $0(0)$ & $0(0)$ & $2(5)$ & \\
\hline Not otherwise specified & $0(0)$ & $0(0)$ & $23(52)$ & \\
\hline PD-L1:TPS, & & & & 0.942 \\
\hline$\geq 50 \%$ & $20(15)$ & $15(34)$ & $17(39)$ & \\
\hline $1-49 \%$ & $20(15)$ & $12(27)$ & $10(23)$ & \\
\hline$<1 \%$ & $16(12)$ & $5(11)$ & $6(14)$ & \\
\hline NA & $75(57)$ & $12(27)$ & $11(25)$ & \\
\hline Line of treatments, & & & & 0.209 \\
\hline $1 \mathrm{st}$ & $7(5)$ & $6(14)$ & $7(16)$ & \\
\hline 2nd & $61(47)$ & $15(34)$ & $22(50)$ & \\
\hline$\geq 3 r d$ & $63(48)$ & $23(52)$ & $15(34)$ & \\
\hline Treatments, & & & & $<0.001$ \\
\hline Nivolumab & $123(94)$ & $37(84)$ & $22(50)$ & \\
\hline Pembrolizumab & $8(6)$ & $7(16)$ & $16(36)$ & \\
\hline Atezolizumab & $0(0)$ & $0(0)$ & $6(14)$ & \\
\hline
\end{tabular}

The data are expressed as number (\%) and median (range)

CNSCLC, common non-small cell lung cancer; ECOG-PS, Eastern Cooperative Oncology Group performance status; LCNEC, large cell neuroendocrine carcinoma; NA, not available; PD-L1, programmed death ligand-1; TPS, tumor proportion score; uNSCLC, uncommon non-small cell lung cancer 
respectively. The histological types were $10(23 \%)$ pleomorphic carcinomas, 9 (20\%) LCNECs, 2 (5\%) large cell carcinomas, and 23 (52\%) NOSs. No patient had epidermal growth factor receptor (EGFR) mutation or anaplastic lymphoma kinase $(A L K)$ fusion. The expression of tumor PD-L1 was TPS $\geq 50 \%$ in 17 (39\%) patients, $1-49 \%$ in $10(23 \%),<1 \%$ in $6(14 \%)$, and not available in $11(25 \%)$. The ICIs were administered as first-, second- or laterline in $7(16 \%), 22(50 \%)$ and $15(34 \%)$ patients, respectively. Of these, 22 (50\%), $16(36 \%)$ and 6 (14\%) patients received nivolumab, pembrolizumab and atezolizumab, respectively. The unmatched $c$ NSCLC group had a significantly lower proportion of men $(p=0.010)$, higher proportion of stage IV disease $(p=0.004)$, better ECOG-PS $(p=0.001)$, and less liver metastasis $(p=0.039)$ compared with the $u$ NSCLC group. A significantly higher proportion of patients in the unmatched $c$ NSCLC group was not evaluated the tumor PD-L1 status $(p<0.001)$. Seven $(5 \%)$ patients and one (1\%) patient in the unmatched $c$ NSCLC group had EGFR gene mutation and $A L K$ fusion gene, respectively. After 1:1 propensity score matching, 44 patients with $c$ NSCLC were selected (matched $c$ NSCLC)
(Fig. 1). The matched $c$ NSCLC group had comparable patients' demographics to the $u$ NSCLC group. Only one patient had an active driver mutation (in EGFR) in the matched $c$ NSCLC group.

In the $u$ NSCLC group, 7 (16\%) patients were still receiving ICIs at the time of data cutoff and the remaining 37 (84\%) were not receiving ICIs because of progressive diseases $(n=27)$, adverse events $(n=8)$ or physician's decision $(\mathrm{n}=2)$ (Fig. 1). In the matched $c$ NSCLC group, only $4(9 \%)$ patients were still receiving ICIs while the other $40(91 \%)$ were not receiving ICIs because of progressive diseases $(n=31)$, adverse events $(n=7)$ or physician's decision $(n=2)$. The median follow-up time was 11.9 months (range 0.1-43.8 months).

\section{Efficacy of ICls}

The ORR of $30 \%$ (95\% confidence interval [CI], 17-45\%) in the $u$ NSCLC group was comparable with $34 \%(95 \%$ CI, $20-50 \%)$ in the matched $c$ NSCLC group $(p=0.819)$ (Fig. 2A) (Table 2). The DCR of 61\% (95\% CI, 45-76\%) in the $u$ NSCLC group was comparable with $61 \%(95 \%$ $\mathrm{CI}, 45-76 \%)$ in the matched $c$ NSCLC group $(p=1.000)$

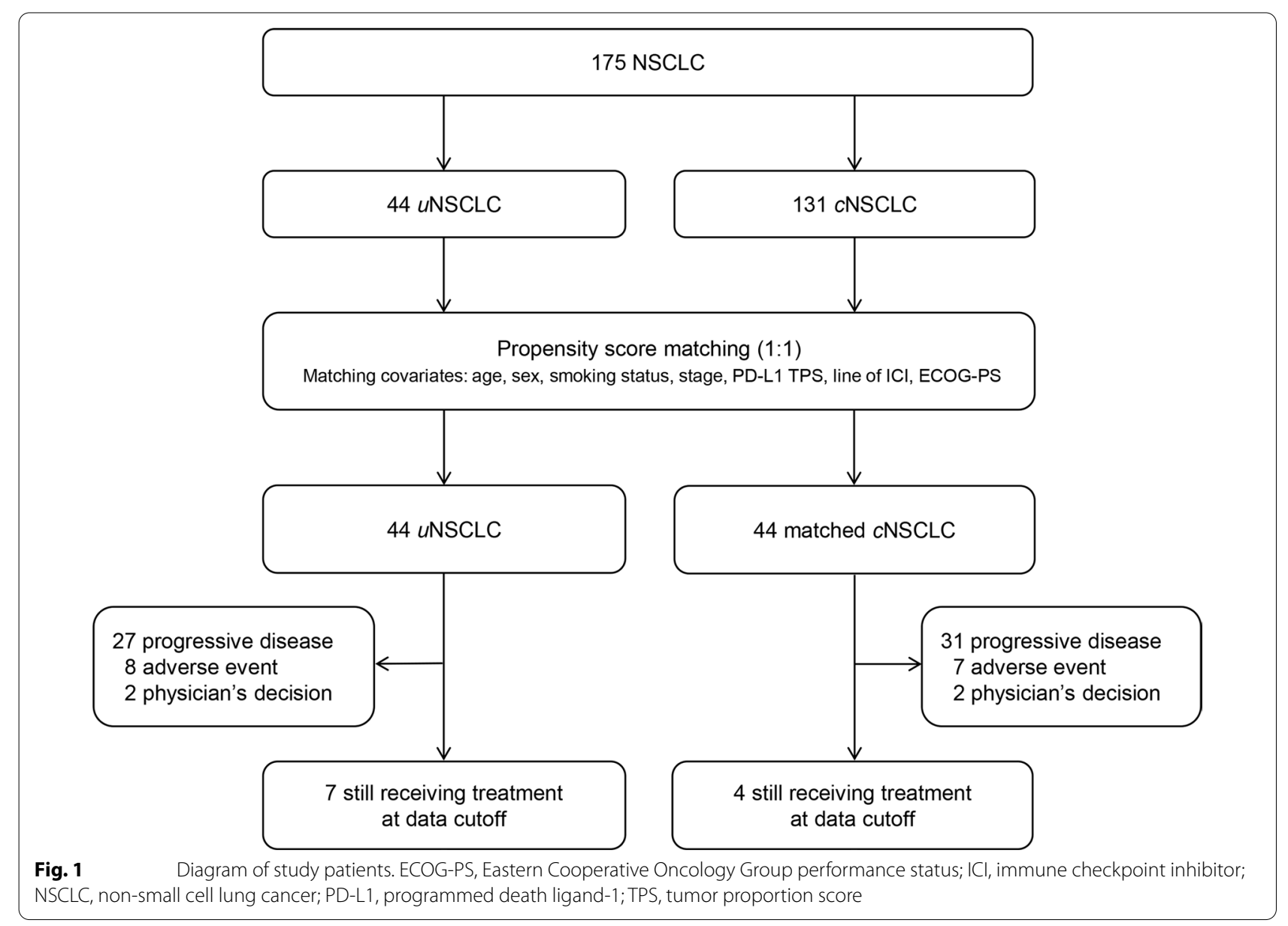



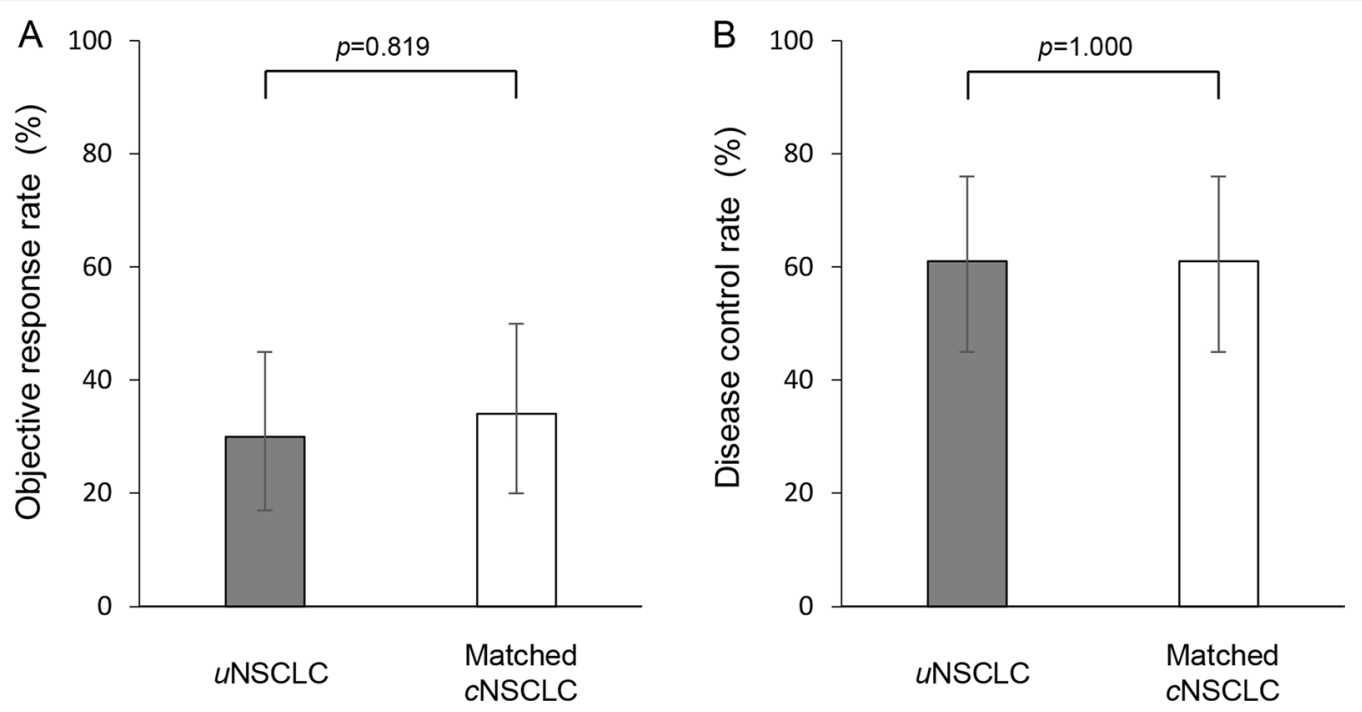

Fig. 2 Objective response rate (ORR) and disease control rate (DCR). (A) ORR and (B) DCR in uncommon non-small cell lung cancer (uNSCLC, gray bar) and propensity-score-matched common NSCLC (CNSCLC, white bar). Error bars indicate $95 \%$ confidence interval

Table 2 Overall response

\begin{tabular}{lllll}
\hline & $\begin{array}{l}\text { Unmatched } \\
\mathbf{c N S C L C} \\
(\mathbf{n = 1 3 1 )}\end{array}$ & $\begin{array}{l}\text { Matched cNSCLC } \\
(\mathbf{n}=\mathbf{4 4 )}\end{array}$ & uNSCLC & $\boldsymbol{p}$-value \\
\hline Response, & & & \\
CR & $0(0)$ & $0(0)$ & $2(5)$ & 0.492 \\
PR & $37(28)$ & $15(34)$ & $11(25)$ & \\
SD & $31(24)$ & $12(27)$ & $14(32)$ & \\
PD & $63(48)$ & $17(39)$ & $17(39)$ & \\
ORR & $28(21-37)$ & $34(20-50)$ & $30(17-45)$ & 0.819 \\
DCR & $52(43-61)$ & $61(45-76)$ & $61(45-76)$ & 1.000 \\
\hline
\end{tabular}

The data are expressed as number (\%) and rate (95\% confidence interval) cNSCLC, common non-small cell lung cancer; $C R$, complete response; $D C R$, disease control rate; $\mathrm{ORR}$, objective response rate; $\mathrm{PD}$, progressive disease; $\mathrm{PR}$, partial response; SD, stable disease; uNSCLC, uncommon non-small cell lung cancer

(Fig. 2B) (Table 2). The median PFS of 4.4 months $(95 \%$ CI 1.8-7.7 months) in the $u$ NSCLC group was not significantly different from 5.4 months (95\% CI 3.1-7.6 months) in the matched $c$ NSCLC group $(p=0.761)$ (Fig. 3A). The median OS of 11.4 months (95\% CI 7.427.4 months) in the $u$ NSCLC group was comparable with 14.1 months (95\% CI 10.6-29.6 months) in the matched $c$ NSCLC group ( $p=0.381)$ (Fig. 3B).

\section{Univariate and multivariate analyses for efficacy of ICls}

In univariate analysis, PD-L1 expression and first-line treatment were predictive for ORR, and ECOG-PS and PD-L1 expression were predictive for DCR (Additional file 1: Tables S1 and S2). In multivariate analysis, PD-L1 expression was predictive for ORR and DCR, and ECOGPS was predictive for ORR (Additional file 1: Tables S1 and S2). In univariate Cox proportional hazard analysis, ever-smokers, ECOG-PS of 0-1, and PD-L1 expression (both $\mathrm{TPS} \geq 50 \%$ and $\geq 1 \%$ ) were significant predictive factors for PFS. In multivariate analysis, ECOG-PS of 0-1 and PD-L1 expression were independent predictive factors for PFS (Table 3). Ever-smokers and ECOG-PS of 0-1 were independent predictive factors for OS in multivariate analysis, while PD-L1 TPS $\geq 50 \%$ demonstrated a borderline predictive significance and TPS $\geq 1 \%$ did not (Table 4). Meanwhile, $u$ NSCLC was not predictive for ORR, DCR, PFS or OS. Additionally, the presence of brain, liver, and bone metastases or line of ICI treatment was not predictive for ORR, DCR, PFS or OS. When limited to the patients with $u$ NSCLC, ECOG-PS of $0-1$ and PD-L1 expression were independent predictive factors for PFS and OS (Additional file 1: Tables S3 and S4).

\section{Subgroup analyses of histological subtypes}

Patients with $u$ NSCLC were evaluated on the basis of histological subtype (Additional file 1: Table S5). In pleomorphic carcinoma, tumor PD-L1 showed TPS $\geq 50 \%$ in $5(50 \%)$ patients, $1-49 \%$ in $3(30 \%)$ patients, and $<1 \%$ in $0(0 \%)$ patients; PD-L1 was not evaluated in 2 patients (20\%). In LCNEC, TPS was $\geq 50 \%$ in 1 (11\%) patient, $1-49 \%$ in $1(11 \%)$ patient, $<1 \%$ in $4(44 \%)$ patients, and not evaluated in 3 (33\%) patients. In NOS, TPS was $\geq 50 \%$ in $9(39 \%)$ patients, $1-49 \%$ in $6(26 \%)$ patients, $<1 \%$ in 2 (9\%) patients, and not evaluated in $6(26 \%)$. The median PFS and OS were 7.7 months (95\% CI: 0.4 months-not estimated [NE]) and 9.5 months (95\% CI: 1.2 


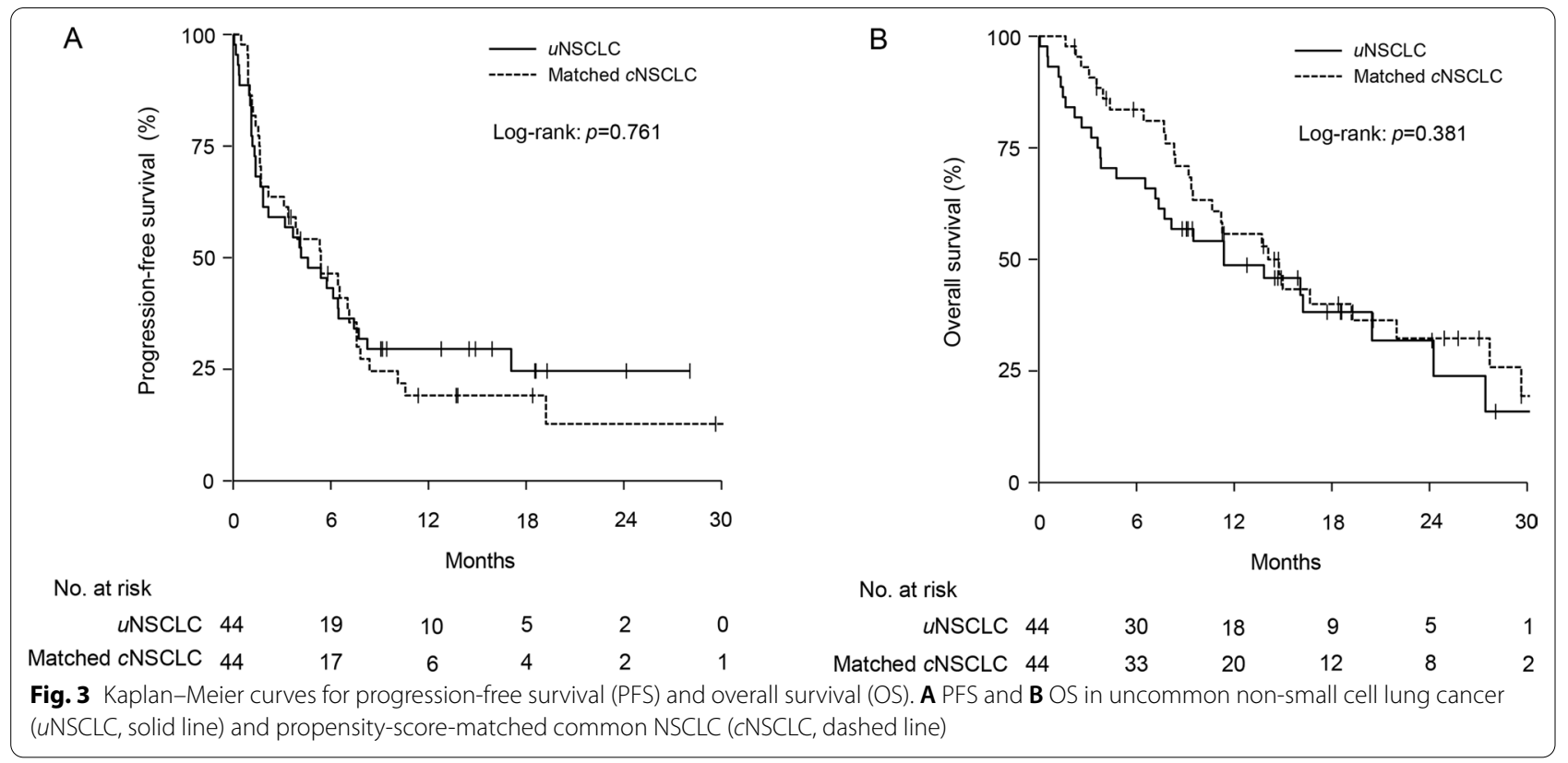

Table 3 Cox proportional hazard analysis for progression-free survival

\begin{tabular}{|c|c|c|c|c|c|c|}
\hline & \multirow{2}{*}{\multicolumn{2}{|c|}{ Univariate }} & \multicolumn{4}{|l|}{ Multivariate } \\
\hline & & & \multicolumn{2}{|l|}{ Set 1} & \multicolumn{2}{|l|}{ Set 2} \\
\hline & $\mathrm{h}(95 \% \mathrm{Cl})$ & $p$-value & $\mathrm{HR}(95 \% \mathrm{Cl})$ & $P$-value & $\mathrm{HR}(95 \% \mathrm{Cl})$ & $p$-value \\
\hline Age, $\geq 65$ & $1.13(0.68-1.87)$ & 0.645 & & & & \\
\hline Sex, men & $1.00(0.31-3.19)$ & 0.996 & & & & \\
\hline Smoking status, ever-smokers & $0.35(0.13-1.00)$ & 0.049 & $0.51(0.17-1.54)$ & 0.232 & $0.35(0.12-1.08)$ & 0.068 \\
\hline ECOG-PS, 0-1 & $0.37(0.19-0.71)$ & 0.003 & $0.42(0.21-0.84)$ & 0.015 & $0.45(0.23-0.89)$ & 0.022 \\
\hline Stage, III & $0.96(0.57-1.62)$ & 0.889 & & & & \\
\hline Pathology, uNSCLC (vs. cNSCLC) & $0.93(0.57-1.50)$ & 0.757 & $1.00(0.61-1.63)$ & 0.996 & $1.01(0.62-1.65)$ & 0.970 \\
\hline \multicolumn{7}{|l|}{ PD-L1, } \\
\hline$\geq 50 \%$ & $0.43(0.25-0.75)$ & 0.003 & $0.44(0.26-0.77)$ & 0.004 & & \\
\hline$\geq 1 \%$ & $0.58(0.36-0.95)$ & 0.029 & & & $0.56(0.33-0.94)$ & 0.029 \\
\hline Line of treatment, 1st-line & $0.81(0.39-1.71)$ & 0.584 & & & & \\
\hline
\end{tabular}

$\mathrm{Cl}$, confidence interval; cNSCLC, common non-small cell lung cancer; ECOG-PS, Eastern Cooperative Oncology Group performance status; HR, hazard ratio; PD-L1, programmed death ligand-1; uNSCLC, uncommon non-small cell lung cancer

months-NE) in pleomorphic carcinoma, respectively; 1.3 months (95\% CI: 0.1 months-NE) and 3.8 months (95\% CI: 0.1 months-NE) in LCNEC; and 4.1 months $(95 \%$ CI: 1.8-6.4 months) and 13.8 months (95\% CI: 6.5-24.2 months) in NOS (Additional file 1: Fig. S1 A-B). No significant difference in PFS and OS was observed in subtypes in the $u$ NSCLC group compared with the matched cNSCLC group.

Patients in the matched $c$ NSCLC were also evaluated separately on the basis of their histological subtypes (Additional file 1: Table S6). The expression of tumor
PD-L1 was TPS $\geq 50 \%$ in $13(41 \%)$ patients, $1-49 \%$ in $8(25 \%),<1 \%$ in $3(9 \%)$, and not evaluated in $8(25 \%)$ in adenocarcinoma; and $\geq 50 \%$ in $2(17 \%), 1-49 \%$ in $4(33 \%),<1 \%$ in $2(17 \%)$, and not evaluated in $4(33 \%)$ in squamous cell carcinoma. The median PFS and OS were 4.2 months (95\% CI: $1.8-7.8$ months) and 16.8 months (95\% CI: 13.7-29.6 months) in adenocarcinoma; and 3.2 months (95\% CI: $1.8-5.3$ months) and 12.5 months (95\% CI: $9.2-19.4$ months) in squamous cell carcinoma (Additional file 1: Fig. S2 A-B). There was no significant difference in PFS $(p=0.132)$ and OS 
Table 4 Cox proportional hazard analysis for overall survival

\begin{tabular}{|c|c|c|c|c|c|c|}
\hline & \multirow{2}{*}{\multicolumn{2}{|c|}{ Univariate }} & \multicolumn{4}{|l|}{ Multivariate } \\
\hline & & & \multicolumn{2}{|l|}{ Set $A$} & \multicolumn{2}{|l|}{ Set B } \\
\hline & $\mathrm{HR}(95 \% \mathrm{Cl})$ & $p$-value & $\mathrm{HR}(95 \% \mathrm{Cl})$ & $P$-value & $\mathrm{HR}(95 \% \mathrm{Cl})$ & $p$-value \\
\hline Age, $\geq 65$ & $1.16(0.67-2.00)$ & 0.606 & & & & \\
\hline Sex, men & $0.80(0.25-2.57)$ & 0.704 & & & & \\
\hline Smoking status, ever-smokers & $0.25(0.09-0.71)$ & 0.009 & $0.30(0.10-0.90)$ & 0.032 & $0.28(0.09-0.84)$ & 0.023 \\
\hline ECOG-PS, 0-1 & $0.34(0.16-0.71)$ & 0.004 & $0.38(0.18-0.82)$ & 0.014 & $0.39(0.19-0.84)$ & 0.015 \\
\hline Stage, III & $0.73(0.41-1.32)$ & 0.298 & & & & \\
\hline Pathology, uNSCLC (vs. cNSCLC) & $1.27(0.75-2.16)$ & 0.381 & $1.29(0.75-2.22)$ & 0.352 & $1.25(0.72-2.16)$ & 0.434 \\
\hline \multicolumn{7}{|l|}{ PD-L1, } \\
\hline$\geq 50 \%$ & $0.61(0.34-1.10)$ & 0.102 & $0.58(0.32-1.06)$ & 0.076 & & \\
\hline$\geq 1 \%$ & $0.88(0.51-1.51)$ & 0.638 & & & $0.80(0.45-1.42)$ & 0.451 \\
\hline Line of treatment, 1st-line & $1.10(0.49-2.46)$ & 0.812 & & & & \\
\hline
\end{tabular}

$\mathrm{Cl}$, confidence interval; cNSCLC, common non-small cell lung cancer; ECOG-PS, Eastern Cooperative Oncology Group performance status; HR, hazard ratio; PD-L1, programmed death ligand-1; uNSCLC, uncommon non-small cell lung cancer

$(p=0.070)$ between adenocarcinoma and squamous cell carcinoma.

\section{Discussion}

In the current study, we found that ICIs were efficacious for patients with $u$ NSCLC and those with $c$ NSCLC with comparable demographic characteristics after propensity score matching. Good ECOG-PS and high PD-L1 expression were significant predictive factors for efficacy of ICIs, regardless of tumor histology. Patients with $u$ NSCLC are known to demonstrate insufficient response to chemotherapy. However, our data indicate that ICIs may provide therapeutic benefits even for patients with $u$ NSCLC, especially those who have good ECOG-PS and high PD-L1 expression.

The median PFS of 4.4 months and median OS of 11.4 months after ICI monotherapy in the current study were comparable with those in previous studies of ICI monotherapy in patients who mostly had $c$ NSCLC (PFS, 2.3-4.0 months and OS, 9.2-13.8 months) [3-6]. In a retrospective study of 21 patients with LCNEC who received ICI monotherapy, median PFS and OS were 4.2 and 11.8 months, respectively [21]. In 49 patients with pulmonary pleomorphic carcinoma who received ICI monotherapy, median PFS and OS were 7.2 and 22.2 months, respectively [22]. Given that conventional chemotherapies for NSCLC often provide limited survival benefits for lung cancer with uncommon histology, ICI monotherapy can be considered as a treatment option [14-16].

Tumor PD-L1 expression is a gold standard biomarker for the efficacy of ICIs in NSCLC; however, the level of tumor PD-L1 expression and its predictive ability varies among different tumor types. For example, only $13.5 \%$ of patients with gastric cancer had PD-L1 TPS $\geq 1 \%$ and the efficacy of nivolumab was not associated with PD-L1 expression [23]. Furthermore, in renal cell carcinoma, $11 \%$ and $24 \%$ of patients had PD-L 1 TPS $\geq 5 \%$ and $\geq 1 \%$, respectively, and the efficacy of nivolumab was not associated with PD-L1 expression [24]. Although $u$ NSCLC has different pathological features from $c$ NCSLC, tumor PD-L1 expression (TPS $\geq 1 \%$ ) was observed in $\sim 60 \%$ of the patients with $u$ NSCLC and was also predictive for efficacy of ICIs.

Good ECOG-PS, a well-known predictive factor for the efficacy of ICIs in NSCLC, was also predictive in $u$ NSCLC $[11,12,25,26]$. Although precise mechanisms underlying ECOG-PS and the efficacy of ICIs are unknown, poor general condition may reflect deteriorated host immune status and lead to weakened effector $\mathrm{T}$ cells. When compared with $c$ NSCLC, $u$ NSCLC tends to progress rapidly and be resistant to standard chemotherapy $[14,15]$. Therefore, it is suggested that patients with $u$ NSCLC are predisposed toward poor general condition without adequate treatments. Approximately $20 \%$ of the patients with $u$ NSCLC had poor ECOG$\mathrm{PS} \geq 2$, compared with only $3 \%$ of those with unmatched $c$ NSCLC. Our data suggest that early initiation of ICIs may be considered for patients with $u$ NSCLC, especially if they have high PD-L1 expression and good ECOG-PS.

There were two main limitations to this study. First, differences in PD-L1 expression and the efficacy of ICIs among different histological subtypes of $u$ NSCLC were unknown, because of the limited number of patients and $25 \%$ of the patients did not undergo PD-L1 testing. It is reported that $80 \%$ of patients with pleomorphic carcinoma had high PD-L1 expression and favorable 
clinical response to ICIs (median PFS 7.2 months and median OS 22.2 months) [22]. Only $10-22 \%$ of patients with LCNEC had PD-L1 expression and had median PFS of 4.2 months and median OS of 11.8 months [21, $27,28]$. In the current study, the patients with pleomorphic carcinoma had the highest proportion of PD-L1 expression and the longest PFS, whereas those with LCNEC had the lowest PD-L1 and the worst PFS. It is possible that the clinical impact of PD-L1 expression and efficacy of ICIs differed owing to the histological subtypes of $u$ NSCLC. Second, we only evaluated ICI monotherapy. Several single or combination therapeutic strategies for ICIs have emerged, such as cytotoxic T-lymphocyte antigen- 4 antibody therapy, combination therapy with ICI and chemotherapy, and combinations of different ICI agents $[17,18,29]$. The clinical benefits of the novel ICIs for $u$ NSCLC are unknown and should be investigated further.

\section{Conclusions}

ICIs had similar clinical efficacy for treatment of $u$ NSCLC and $c$ NSCLC. Additionally, good ECOG-PS and high PD-L1 expression were predictive for the efficacy of ICIs in $u$ NSCLC.

\section{Supplementary Information}

The online version contains supplementary material available at https://doi. org/10.1186/s12890-021-01681-6.

Additional file 1. Table S1. Logistic regression analysis for objective response. Table S2. Logistic regression analysis for disease control rate. Table S3. Cox proportional hazard analysis for progression-free survival in uncommon non-small cell lung cancer. Table S4. Cox proportional hazard analysis for overall survival in uncommon non-small cell lung cancer. Table S5. Patient characteristics according to histological subtypes in uncommon non-small cell lung cancer. Table S6. Patient characteristics according to histological subtypes in matched common non-small cell lung cancer. Fig. S1. Kaplan-Meier curves for progression-free survival and overall survival by histology in uncommon non-small cell lung cancer group. Fig. S2. Kaplan-Meier curves for progression-free survival and overall survival by histology in matched common non-small cell lung cancer group.

\section{Acknowledgements}

We would like to acknowledge patients and their families. We thank Cathel Kerr, BSc, PhD, from Edanz (https://jp.edanz.com/ac) for editing a draft of this manuscript.

\section{Authors' contributions}

KM has full access to all of the data in the study and takes responsibility for the integrity of the data and the accuracy of the data analysis. MK participated in study concept and design; acquisition, analysis and interpretation of data; statistical analyses; and drafting of the manuscript. NI participated in study concept and design, interpretation of data, drafting of the manuscript, and supervision. Yl, HH, YS, KF, TF, NE and YN participated in study concept and design, interpretation of data, and drafting of the manuscript. MK, TM, MN, KK, MT, SM, SM, KA, MF, HK and HM participated in study concept and design, data acquisition, and drafting of the manuscript. TS participated in study concept and design, drafting of the manuscript, and supervision. All authors read and approved the final manuscript.

\section{Funding}

This research did not receive any specific grant from funding agencies in the public, commercial, or not-for-profit sectors.

\section{Availability of data and materials}

The datasets used and/or analyzed during the current study are available from the corresponding author on reasonable request.

\section{Declarations}

\section{Ethics approval and consent to participate}

The study protocol was approved by the Institutional Review Board of Hamamatsu University School of Medicine (No. 19-122).

\section{Consent to participate}

Not applicable.

\section{Competing interests}

All authors have no conflict of interest in relation to this manuscript.

\section{Author details}

${ }^{1}$ Second Division, Department of Internal Medicine, Hamamatsu University School of Medicine, 1-20-1 Handayama, Hamamatsu 431-3192, Japan. ${ }^{2}$ Department of Chemotherapy, Hamamatsu University School of Medicine, 1-20-1 Handayama, Hamamatsu 431-3192, Japan. ${ }^{3}$ Department of Respiratory Medicine, Seirei Hamamatsu General Hospital, 2-12-12 Sumiyoshi, Hamamatsu 430-8558, Japan. ${ }^{4}$ Department of Respiratory Medicine, Seirei Mikatahara General Hospital, 3453 Mikatahara-cho, Hamamatsu 433-8558, Japan. ${ }^{5}$ Department of Respiratory Medicine, Hamamatsu Medical Center, 328 Tomitsuka-cho, Hamamatsu 432-8580, Japan. ${ }^{6}$ Department of Respiratory Medicine, Hamamatsu Rosai Hospital, 25 Shougen-cho, Hamamatsu 430-8525, Japan. ${ }^{7}$ Department of Respiratory Medicine, Iwata City Hospital, 513-2 Ohkubo, Iwata 438-8550, Japan. ${ }^{8}$ Department of Respiratory Medicine, Fujieda Municipal General Hospital, 4-1-11 Surugadai, Fujieda 426-8677, Japan. ${ }^{9}$ Department of Respiratory Medicine, Shizuoka General Hospital, 4-27-1 Kita-ando, Shizuoka 420-0881, Japan. ${ }^{10}$ Department of Respiratory Medicine, Shizuoka City Hospital, 10-93 Ote-cho, Shizuoka 420-8630, Japan. ${ }^{11}$ Department of Respiratory Medicine, Shizuoka Saiseikai Hospital, 1-1-1 Oshika, Shizuoka 422- 8527, Japan. ${ }^{12}$ Department of Respiratory Medicine, Japanese Red Cross Shizuoka Hospital, 8-2 Otemachi, Shizuoka 420-0853, Japan. ${ }^{13}$ Department of Clinical Pharmacology and Therapeutics, Hamamatsu University School of Medicine, 1-20-1 Handayama, Hamamatsu 431-3192, Japan.

Received: 3 June 2021 Accepted: 29 September 2021

Published online: 02 October 2021

\section{References}

1. Reck M, Rodriguez-Abreu D, Robinson AG, et al. Pembrolizumab versus chemotherapy for PD-L1-positive non-small-cell lung cancer. N Engl J Med. 2016:375:1823-33.

2. Herbst RS, Giaccone G, de Marinis F, et al. Atezolizumab for first-line treatment of PD-L1-selected patients with NSCLC. N Engl J Med. 2020;383:1328-39.

3. Brahmer J, Reckamp KL, Baas P, et al. Nivolumab versus docetaxel in advanced squamous-cell non-small-cell lung cancer. N Engl J Med. 2015:373:123-35

4. Borghaei $H$, Paz-Ares $L$, Horn L, et al. Nivolumab versus docetaxel in advanced nonsquamous non-small-cell lung cancer. N Engl J Med. 2015;373:1627-39.

5. Herbst RS, Baas P, Kim DW, et al. Pembrolizumab versus docetaxel for previously treated, PD-L1-positive, advanced non-small-cell lung cancer (KEYNOTE-010): a randomised controlled trial. Lancet. 2016;387:1540-50.

6. Rittmeyer A, Barlesi F, Waterkamp D, et al. Atezolizumab versus docetaxel in patients with previously treated non-small-cell lung cancer (OAK): a 
phase 3, open-label, multicentre randomised controlled trial. Lancet. 2017;389:255-65.

7. National Comprehensive Cancer Network, NCCN Clinical Practice Guidelines in Oncology (NCCN Guidelines), non-small cell lung cancer. version 4. 2021. https://www.nccn.org/professionals/physician_gls/pdf/nscl.pdf. Accessed 16 May 2021.

8. Planchard D, Popat S, Kerr K, et al. Metastatic non-small cell lung cancer: ESMO Clinical Practice Guidelines for diagnosis, treatment and follow-up. Ann Oncol. 2018:29:iv192-237.

9. Hanna NH, Schneider BJ, Temin S, et al. Therapy for stage IV non-small-cell lung cancer without driver alterations: ASCO and $\mathrm{OH}$ (CCO) joint guideline update. J Clin Oncol. 2020;38:1608-32.

10. Yang P, Allen MS, Aubry MC, et al. Clinical features of 5,628 primary lung cancer patients: experience at Mayo Clinic from 1997 to 2003. Chest. 2005;128:452-62.

11. Lin SY, Yang CY, Liao BC, et al. Tumor PD-L1 expression and clinical outcomes in advanced-stage non-small cell lung cancer patients treated with nivolumab or pembrolizumab: real-world data in Taiwan. J Cancer. 2018:9:1813-20

12. Morita R, Okishio K, Shimizu J, et al. Real-world effectiveness and safety of nivolumab in patients with non-small cell lung cancer: a multicenter retrospective observational study in Japan. Lung Cancer. 2020;140:8-18.

13. Travis WD, Brambilla E, Nicholson AG, et al. The 2015 World Health Organization classification of lung tumors: impact of genetic, clinical and radiologic advances since the 2004 classification. J Thorac Oncol. 2015;10:1243-1260

14. Vieira T, Girard N, Ung M, et al. Efficacy of first-line chemotherapy in patients with advanced lung sarcomatoid carcinoma. J Thorac Oncol. 2013:8:1574-7.

15. Righi L, Vavala T, Rapa I, et al. Impact of non-small-cell lung cancer-not otherwise specified immunophenotyping on treatment outcome. J Thorac Oncol. 2014;9:1540-6.

16. Sun JM, Ahn MJ, Ahn JS, et al. Chemotherapy for pulmonary large cell neuroendocrine carcinoma: similar to that for small cell lung cancer or non-small cell lung cancer? Lung Cancer. 2012;77:365-70.

17. Socinski MA, Jotte RM, Cappuzzo F, et al. Atezolizumab for firstline treatment of metastatic nonsquamous NSCLC. N Engl J Med. 2018:378:2288-301.

18. Gandhi L, Rodriguez-Abreu D, Gadgeel S, et al. Pembrolizumab plus chemotherapy in metastatic non-small-cell lung cancer. N Engl J Med. 2018;378:2078-92.
19. Eisenhauer EA, Therasse P, Bogaerts J, et al. New response evaluation criteria in solid tumours: revised RECIST guideline (version 1.1). Eur J Cancer. 2009:45:228-47.

20. Kanda Y. Investigation of the freely available easy-to-use software'EZR' for medical statistics. Bone Marrow Transplant. 2013;48:452-8.

21. Sherman S, Rotem O, Shochat T, Zer A, Moore A, Dudnik E. Efficacy of immune check-point inhibitors (ICPi) in large cell neuroendocrine tumors of lung (LCNEC). Lung Cancer. 2020;143:40-6.

22. Lee J, Choi Y, Jung HA, et al. Outstanding clinical efficacy of PD-1/ PD-L1 inhibitors for pulmonary pleomorphic carcinoma. Eur J Cancer. 2020:132:150-8.

23. Kang YK, Boku N, Satoh T, et al. Nivolumab in patients with advanced gastric or gastro-oesophageal junction cancer refractory to, or intolerant of, at least two previous chemotherapy regimens (ONO-4538-12, ATTRAC TION-2): a randomised, double-blind, placebo-controlled, phase 3 trial. Lancet. 2017;390:2461-71.

24. Motzer RJ, Escudier B, McDermott DF, et al. Nivolumab versus everolimus in advanced renal-cell carcinoma. N Engl J Med. 2015:373:1803-13.

25. Juergens RA, Mariano C, Jolivet J, et al. Real-world benefit of nivolumab in a Canadian non-small-cell lung cancer cohort. Curr Oncol. 2018;25:384-92.

26. Crinò L, Bidoli P, Delmonte A, et al. Italian cohort of nivolumab expanded access program in squamous non-small cell lung cancer: Results from a real-world population. Oncologist. 2019;24:e1165-71.

27. Eichhorn F, Harms A, Warth A, Muley T, Winter H, Eichhorn ME. PD-L1 expression in large cell neuroendocrine carcinoma of the lung. Lung Cancer. 2018;118:76-82

28. Tsuruoka K, Horinouchi H, Goto Y, et al. PD-L1 expression in neuroendocrine tumors of the lung. Lung Cancer. 2017;108:115-20.

29. Hellmann MD, Paz-Ares L, Bernabe Caro R, et al. Nivolumab plus Ipilimumab in advanced non-small-cell lung cancer. N Engl J Med. 2019;381:2020-31.

\section{Publisher's Note}

Springer Nature remains neutral with regard to jurisdictional claims in published maps and institutional affiliations.
Ready to submit your research? Choose BMC and benefit from:

- fast, convenient online submission

- thorough peer review by experienced researchers in your field

- rapid publication on acceptance

- support for research data, including large and complex data types

- gold Open Access which fosters wider collaboration and increased citations

- maximum visibility for your research: over $100 \mathrm{M}$ website views per year

At BMC, research is always in progress.

Learn more biomedcentral.com/submissions 